\title{
High-Resolution Surface Analysis of Mammalian Sperm Head Substructures
}

\author{
Angela V. Klaus
}

Microscopy and Imaging Facility, American Museum of Natural History, New York, NY 10024

Mammalian spermatozoa are highly differentiated cells that are morphologically and biochemically unique. Following the second meiotic division, sperm cells undergo a remarkable transformation from haploid round spermatids to elongate spermatozoa in a process called spermiogenesis [for review see 1]. During this process, the nucleus elongates, flattens, and assumes a species-specific shape. The acrosome forms over the apical portion of the sperm head, and the sperm tail forms caudally. The chromatin condenses into a highly compacted, transcriptionally silent mass, and a proteinaceous layer of material termed the perinuclear theca is deposited in close apposition to the cytoplasmic side of the nuclear envelope. Spermiogenesis takes places within the seminiferous epithelium of the testis; final sperm maturation occurs within the lumen of the epididymis.

Ultrastructural characteristics of mature spermatozoa have mainly been studied using thin sectioning and transmission electron microscopy (TEM) [for example see 2]. Other studies have used TEM replicas in order to provide surface views of sperm structures [for example see 3]. The current work is an alternative approach for visualizing sperm substructures. Here, the structural and biochemical nature of the mature sperm head was interrogated by perturbation with detergents and reducing agent, followed by visualization with cold-field emission scanning electron microscopy (FE-SEM). The high-resolution surface images obtained in this manner revealed structures of the mammalian sperm head that would be difficult to visualize using conventional techniques.

Methods and Results:

Samples of rodent sperm were isolated from cauda epididymides and briefly treated with nonionic, anionic, or cationic detergent (Triton X-100, SDS or ATAB, respectively), with or without reducing agent (DTT), in order to visualize substructures of the perinuclear theca (PT). Other samples were treated sequentially with ATAB and DTT in order to completely remove the PT [4]. PT-depleted sperm were then treated with DNase to investigate substructures of the sperm nucleus. Treated sperm were immobilized on poly-L-lysine-coated coverslips, fixed, critical point dried, sputter-coated and viewed using an Hitachi S-4700 cold-field emission SEM. The images in Figs. 1 and 2 show representative structures detected using the above methods for mouse and hamster sperm, respectively.

References

[1] Y. Clermont et al., Cell and Molecular Biology of the Testis, (eds. Desjardins, C. \& Ewing, L.L.), Oxford Univ. Press, New York, 1993

[2] R. Yanagimachi and Y.D. Noda, Am. J. Anat. 128 (1970) 367

[3] D.M. Phillips, J. Exp. Zool. 191 (1975) 8

[4] S.B. Moss et al., Mol. Reprod. Dev. 34 (1993) 164. 

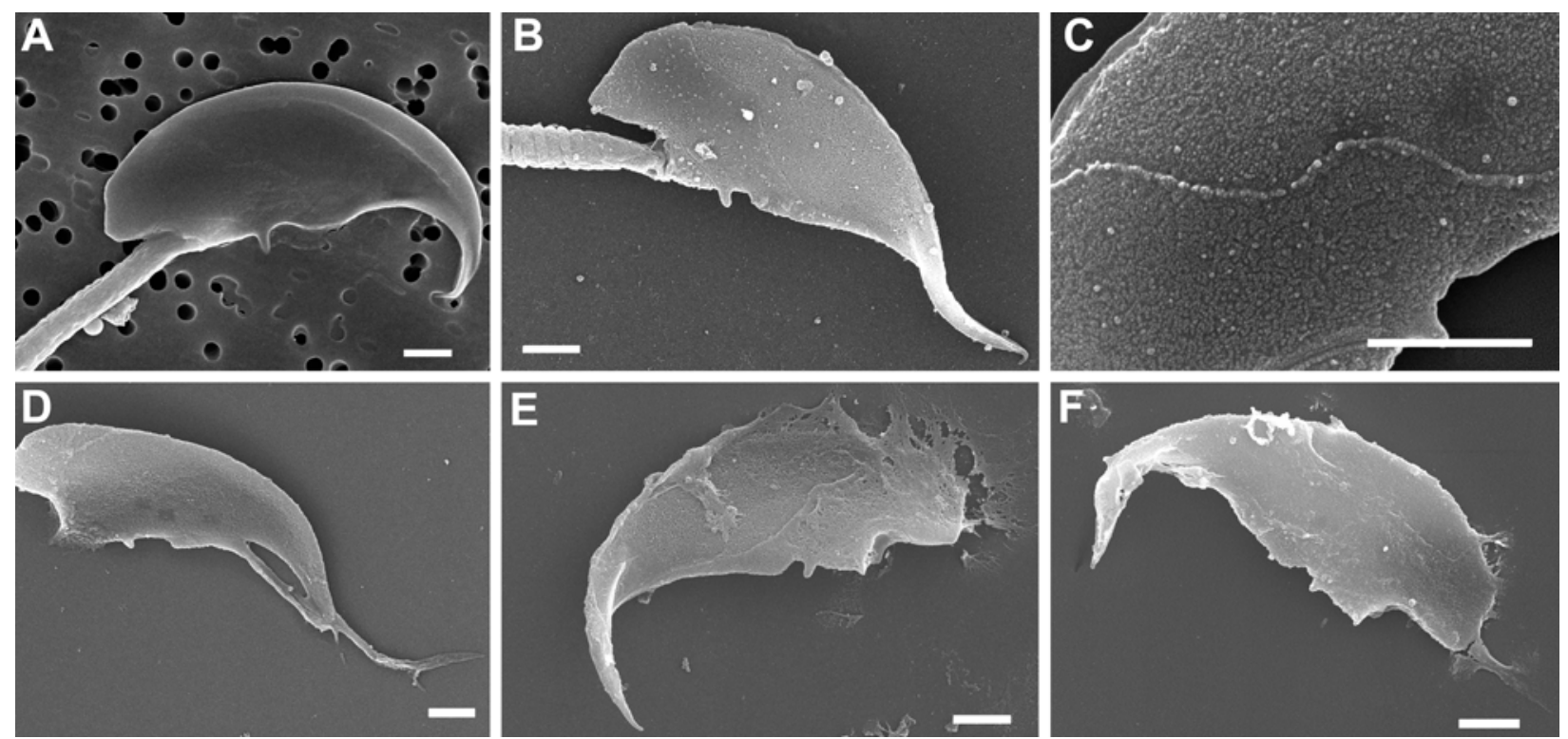

Fig. 1. Mouse epididymal sperm briefly treated with detergents and reducing agent. (A) Intact, untreated sperm. (B) Triton X-100 only; acrosomal and plasma membranes have been removed, PT is intact. (C) SDS only; higher magnification image of the equatorial region showing a detergent-resistant structure (postacrosomal ridge). (D) SDS + DTT; rod-like structures within the PT are apparent. (E) ATAB only; PT disrupted, portions still intact. (F) ATAB + DTT; PT disrupted, portions still intact. Bar $=1$ micron for all panels.
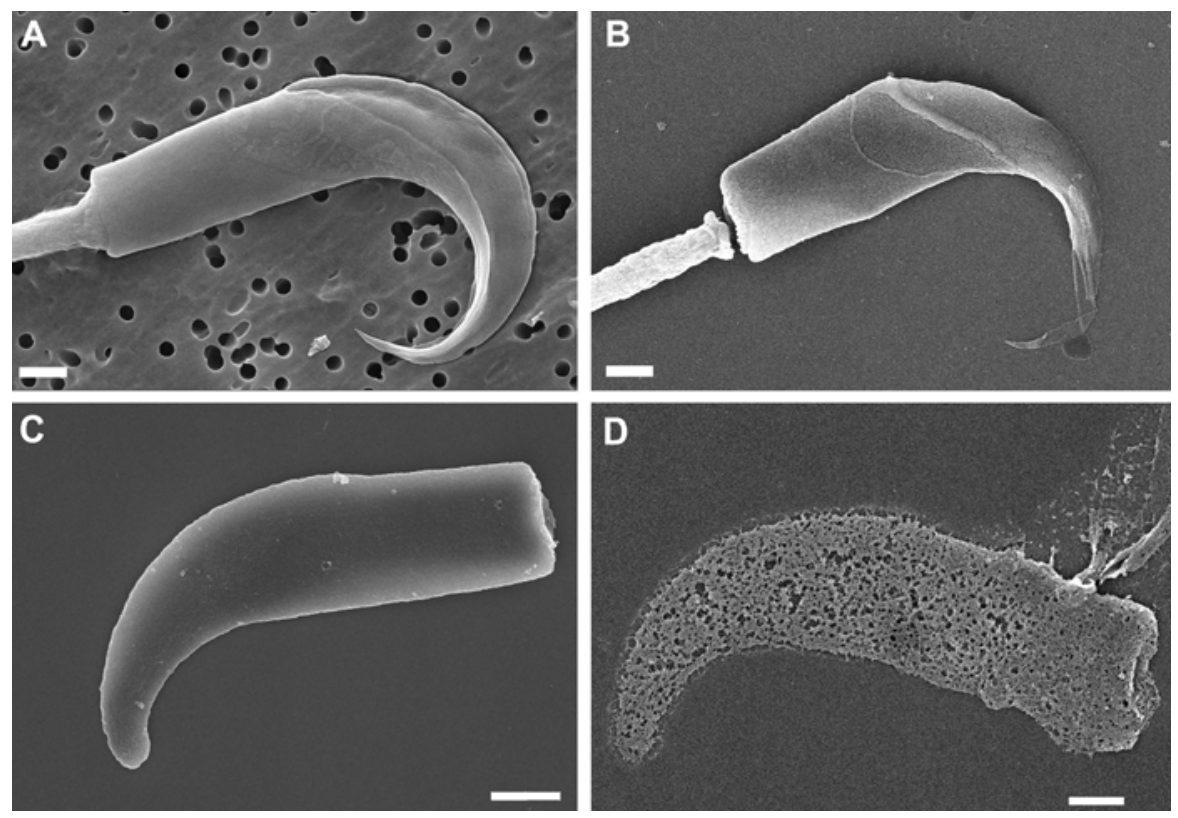

Fig. 2. Hamster epididymal sperm. (A) Intact, untreated sperm. (B) Triton X-100 only; PT intact. (C) ATAB, DTT treatment; PT-depleted. (D) PT-depleted sperm head treated with DNase. Bar $=1$ micron for all panels. 